\title{
Flat coordinates and dilaton fields for three-dimensional conformal sigma models
}

\author{
Ladislav Hlavatý and Miroslav Turek \\ Faculty of Nuclear Sciences and Physical Engineering, \\ Czech Technical University \\ Břehová 7, 11519 Prague 1, Czech Republic \\ e-mail: hlavaty@fjfi.cvut.cz, turekm@km1.fjif.cvut.cz
}

August 27, 2018

\begin{abstract}
Riemannian coordinates for flat metrics corresponding to three-dimensional conformal Poisson-Lie T-dualizable sigma models are found by solving partial differential equations that follow from the transformations of the connection components. They are then used for finding general forms of the dilaton fields satisfying the vanishing beta equations of the sigma models.
\end{abstract}

\section{Introduction}

Recent interest in the conformal invariant $\sigma$-models follows from their relation to the string theory. Principal $\sigma$-model can be defined as a field theory on a Lie group $G$ on which a covariant second order tensor field $F$ is given. The action of the $\sigma$-model then is

$$
S_{F}[\phi]=\int d^{2} x \partial_{-} \phi^{i} F_{i j}(\phi) \partial_{+} \phi^{j}
$$

where the functions $\phi^{j}: \mathbf{R}^{2} \rightarrow \mathbf{R}, j=1,2, \ldots, \operatorname{dim} G$ are obtained by the composition $\phi^{j}=y^{j} \circ \phi$ of a map $\phi: \mathbf{R}^{2} \rightarrow G$ and a coordinate map $y: U_{g} \rightarrow \mathbf{R}^{n}, n=\operatorname{dim} G$ of a neighborhood of an element $\phi\left(x_{+}, x_{-}\right)=g \in G$.

The equations of motion have the form

$$
\partial_{-} \partial_{+} \phi^{j}+\gamma_{r s}^{j} \partial_{-} \phi^{r} \partial_{+} \phi^{s}=0
$$

where

$$
\gamma_{r s}^{j}:=\frac{1}{2} G^{j i}\left(F_{i s, r}+F_{r i, s}-F_{r s, i}\right)
$$

and $G^{j i}$ is the inverse of

$$
G_{i j}=\frac{1}{2}\left(F_{i j}+F_{j i}\right)
$$

Quantization of the $\sigma$-models requires that they be made conformal invariant. This is achieved by addition of another term depending on a scalar (dilaton) field $\Phi$ to the action (11). To guarantee the conformal invariance of the $\sigma$-model (at least at the one-loop level) 
the fields $F$ and $\Phi$ must satisfy the so called vanishing $\beta$ equations

$$
\begin{aligned}
0 & =R_{i j}-\nabla_{i} \nabla_{j} \Phi-\frac{1}{4} H_{i m n} H_{j}^{m n} \\
0 & =\nabla^{k} \Phi H_{k i j}+\nabla^{k} H_{k i j}, \\
0 & =R-2 \nabla_{k} \nabla^{k} \Phi-\nabla_{k} \Phi \nabla^{k} \Phi-\frac{1}{12} H_{k m n} H^{k m n},
\end{aligned}
$$

where covariant derivatives $\nabla_{k}$, Ricci tensor $R_{i j}$ and scalar curvature $R$ are calculated from the (pseudo)metric (44) that is also used for lowering and raising indices. The components of torsion are defined as

$$
H_{i j k}=\partial_{i} B_{j k}+\partial_{j} B_{k i}+\partial_{k} B_{i j}
$$

where

$$
B_{i j}=\frac{1}{2}\left(F_{i j}-F_{j i}\right)
$$

We shall be interested in $\sigma$-models that satisfy the vanishing $\beta$ equations and moreover are Poisson-Lie T-dualizable i.e. satisfy [2]

$$
\mathcal{L}_{v_{i}}(F)_{\mu \nu}=F_{\mu \kappa} v_{j}^{\kappa} \tilde{f}_{i}^{j k} v_{k}^{\lambda} F_{\lambda \nu}, i=1, \ldots, \operatorname{dim} G,
$$

where $v_{i}$ form a basis of left-invariant fields on $G$ and $\tilde{f}_{i}^{j k}$ are structure coefficients of a Lie group $\tilde{G}$ such that $\operatorname{dim} \tilde{G}=\operatorname{dim} G$. If $F$ satisfies the equation (10) then the equations of motion of the $\sigma$-model can be rewritten (see 1], 2]) as equations for maps to the sixdimensional Drinfel'd double $D=(G \mid \tilde{G})$ - connected Lie group whose Lie algebra $\mathcal{D}$ admits a decomposition

$$
\mathcal{D}=\mathcal{G}+\tilde{\mathcal{G}}
$$

into two subalgebras that are maximally isotropic with respect to a bilinear, symmetric, nondegenerate, ad-invariant form on $\mathcal{D}$.

There are two important types of coordinates on the manifolds where the $\sigma$-models live. The first one is given by the Lie group structure and follows from the possibility to express the elements of the Lie group (at least in the vicinity of the unit) as a product of elements of one-parametric subgroups. The Poisson-Lie T-dual $\sigma$-models are usually expressed in terms of these group coordinates. The other type of coordinates are those in which the metric on the manifold have a special simple form. They are called Riemannian coordinates (see e.g. 3]). The Riemannian coordinates of the flat metrics will be called flat coordinates here. In these coordinates the flat metric tensors become constant and the Christoffel symbols vanish so that the equations of motion (2) as well as the vanishing $\beta$ equations (5)-(7) become very simple. That's why it is very desirable to find the transformation between the group and Riemannian coordinates of the $\sigma$-models.

\section{Investigated models}

In the paper [4] the semiabelian Drinfel'd doubles $(G \mid 1)$, for which $\mathcal{G}$ in the decomposition (11) are solvable Bianchi algebras $\mathbf{2}, \mathbf{3}, \mathbf{4}, \mathbf{5}, \mathbf{6}_{\mathbf{0}}, \mathbf{7}_{\mathbf{0}}$ and $\tilde{\mathcal{G}}$ is the three-dimensional Abelian Lie algebra 1 (for the notation see [4], [5]), were investigated and classification of conformal invariant Poisson-Lie T-dualizable $\sigma$-models with constant dilaton field was done. A bit surprisingly, all these models are not only Ricci flat and torsionless but also flat in the sense that their Riemann-Christoffel tensor vanishes.

The metrics are expressed in the group coordinates $y_{1}, y_{2}, y_{3}$, for which elements of the group $G$ are parametrized as (for typographic reasons we use subscripts for coordinates in the following)

$$
g(y)=e^{y_{1} X_{1}} e^{y_{2} X_{2}} e^{y_{3} X_{3}},
$$


where $X_{1}, X_{2}, X_{3}$ are generators of the corresponding Lie algebra, and we are going to find the transformations of the group coordinates to the flat coordinates. The metrics corresponding to the investigated Drinfel'd doubles are

$(2 \mid 1)$ :

$$
G(y)_{i j}=\left(\begin{array}{ccc}
0 & u & v \\
u & q & g+u y_{2} \\
v & g+u y_{2} & r+2 v y_{2}
\end{array}\right)
$$

$(3 \mid 1):$

$$
G(y)_{i j}=\left(\begin{array}{ccc}
p & u+z e^{-2 y_{1}} & -u+z e^{-2 y_{1}} \\
u+z e^{-2 y_{1}} & q & -q \\
-u+z e^{-2 y_{1}} & -q & q
\end{array}\right),
$$

$(4 \mid 1)$ :

$$
G(y)_{i j}=\left(\begin{array}{ccc}
p & \left(v y_{1}+u\right) e^{-y_{1}} & v e^{-y_{1}} \\
\left(v y_{1}+u\right) e^{-y_{1}} & q e^{-2 y_{1}} & 0 \\
v e^{-y_{1}} & 0 & 0
\end{array}\right)
$$

$(5 \mid 1):$

$$
G(y)_{i j}=\left(\begin{array}{ccc}
p & u e^{-y_{1}} & v e^{-y_{1}} \\
u e^{-y_{1}} & \frac{g^{2}}{r} e^{-2 y_{1}} & g e^{-2 y_{1}} \\
v e^{-y_{1}} & g e^{-2 y_{1}} & r e^{-2 y_{1}}
\end{array}\right)
$$

$\left(6_{0} \mid 1\right):$

$$
G(y)_{i j}=\left(\begin{array}{ccc}
p & 0 & v+p y_{2} \\
0 & -p & g-p y_{1} \\
v+p y_{2} & g-p y_{1} & r+2 g y_{1}+2 v y_{2}+p\left(y_{2}^{2}-y_{1}^{2}\right)
\end{array}\right)
$$

$\left(7_{0} \mid 1\right):$

$$
G(y)_{i j}=\left(\begin{array}{ccc}
p & 0 & z+p y_{2} \\
0 & p & g-p y_{1} \\
z+p y_{2} & g-p y_{1} & r-2 g y_{1}+2 z y_{2}+p\left(y_{1}^{2}+y_{2}^{2}\right)
\end{array}\right)
$$

where $u, v, p, q, g, r, z$ are arbitrary real constants.

Beside these models, solutions of the vanishing $\beta$ equations with flat metrics and nonconstant dilaton fields $\Phi$ were found by the Poisson-Lie T-duality on the Drinfel'd double $\left(1 \mid 6_{0}\right) \cong\left(5 i i \mid 6_{0}\right) \cong\left(1 \mid 6_{0}\right)$. The metrics and the dilaton fields expressed in the group coordinates read

$$
\begin{aligned}
\left(1 \mid 6_{0}\right): & \\
G(y)_{i j} & =K\left(y_{1}, y_{2}\right)^{-1}\left(\begin{array}{ccc}
-k^{2} q y_{1}^{2} & k^{2} q y_{1} y_{2} & -k\left(1+k y_{1}\right) \\
k^{2} q y_{1} y_{2} & q\left(-1+k^{2} y_{2}{ }^{2}\right) & k^{2} y_{2} \\
-k\left(1+k y_{1}\right) & k^{2} y_{2} & 0
\end{array}\right), \\
\Phi & =\ln \mid\left(K\left(y_{1}, y_{2}\right) \mid+C,\right.
\end{aligned}
$$

where $k, q$ are constants and

$$
K\left(y_{1}, y_{2}\right)=1+2 k y_{1}+k^{2}\left(y_{1}^{2}-y_{2}^{2}\right) .
$$

$\left(5 i i \mid 6_{0}\right)$ : 


$$
\begin{aligned}
G(y)_{11} & =\frac{q\left(w^{2}-1\right)}{4 W\left(y_{1}, y_{2}\right)}\left(1+e^{2 y_{1}+2 y_{2}}-2 e^{2 y_{1}+y_{2}}\right)^{2}, \\
G(y)_{21} & =\frac{q}{4 W\left(y_{1}, y_{2}\right)}\left(1-2 e^{2 y_{1}+y_{2}}+e^{2 y_{1}+y_{2}}\right)\left(w^{2}\left(1-2 e^{y_{1}}+e^{2 y_{1}+2 y_{2}}\right)-1-e^{2 y_{1}+2 y_{2}}\right), \\
G(y)_{22} & =\frac{q}{4 W\left(y_{1}, y_{2}\right)}\left(w^{2}\left(1-2 e^{y_{1}}+e^{2 y_{1}+2 y_{2}}\right)^{2}-\left(1+e^{2 y_{1}+2 y_{2}}\right)^{2}\right), \\
G(y)_{31} & =\frac{w}{2 W\left(y_{1}, y_{2}\right)} e^{y_{1}+y_{2}}\left(\left(2 e^{2 y_{1}+y_{2}}-e^{2 y_{1}+2 y_{2}}\right)(w-1)-w-1\right), \\
G(y)_{32} & =\frac{w}{2 W\left(y_{1}, y_{2}\right)} e^{y_{1}+y_{2}}\left(2 w e^{y_{1}}-e^{2 y_{1}+2 y_{2}}(w-1)-w-1\right), \\
G(y)_{33} & =0 \\
\Phi & =\ln \left|(1+w) e^{-\left(y_{1}+y_{2}\right)}+w\left(1-2 e^{-y_{2}}\right)\right|+\ln \left|(w-1) e^{y_{1}+y_{2}}-w\right|+C,
\end{aligned}
$$

where $w$ is a constant and

$$
W\left(y_{1}, y_{2}\right)=e^{y_{1}+y_{2}}\left((w-1) e^{y_{1}+y_{2}}-w\right)\left(1+w-2 w e^{y_{1}}+w e^{y_{1}+y_{2}}\right) .
$$

The investigated models can also have nonzero antisymmetric part $B$ of the tensor $F$ but the corresponding torsions $H_{i j k}$ given by (8) are zero so that we assume that $F_{i j}=G_{i j}$ in the following. In spite of the fact that all the metrics above are flat, the task to find coordinates for which the metrics become constant is not trivial.

\section{Flat coordinates}

For finding the flat coordinates we shall use the formula for transformation of the Levi-Civita connection

$$
\Gamma_{j k}^{i}(y)=\frac{1}{2} G^{l i}\left(\frac{\partial G_{k l}}{\partial y_{j}}+\frac{\partial G_{j l}}{\partial y_{k}}-\frac{\partial G_{k j}}{\partial y_{l}}\right) .
$$

that reads as

$$
\Gamma_{j k}^{i}(y)=\frac{\partial y_{i}}{\partial \xi^{l}} \frac{\partial \xi_{m}}{\partial y_{j}} \frac{\partial \xi_{n}}{\partial y_{k}} \Gamma_{m n}^{\prime l}(\xi)+\frac{\partial y_{i}}{\partial \xi_{l}} \frac{\partial^{2} \xi_{l}}{\partial y_{j} \partial y_{k}} .
$$

The components of $\Gamma_{m n}^{\prime l}(\xi)$ in the flat coordinates vanish and we get the system of partial differential equations for $\xi(y)$

$$
\frac{\partial^{2} \xi_{i}}{\partial y_{j} \partial y_{k}}=\Gamma_{j k}^{l} \frac{\partial \xi_{i}}{\partial y_{l}}
$$

The system is linear and moreover separated with respect to the unknowns $\xi_{i}$ 's. The possibility to solve it explicitly depends on the form of $\Gamma_{j k}^{l}$. We were able to find general explicit solutions for the metrics given above that together with the suitable initial conditions will produce the Riemannian coordinates. The initial condition

$$
\left[\frac{\partial \xi_{k}}{\partial y_{i}}\right]_{\vec{y}=\overrightarrow{0}}=\delta_{k}^{i}
$$

produce the coordinates in which the metric acquires the constant form $\tilde{G}(\xi)=G(y=0)$ that can be further diagonalized.

In the following we shall present solution of the equations (25) in detail for the metric (17) and write down the results for the other metrics. 


\subsection{Flat coordinates for the $\sigma$-model on $\left(6_{0} \mid 1\right)$}

The nonzero components of the affine connection for the metric (17) are

$$
\begin{array}{ll}
\Gamma_{23}^{1}=1, & \Gamma_{33}^{1}=\frac{-g+p y_{1}}{p}, \\
\Gamma_{13}^{2}=1, & \Gamma_{33}^{2}=\frac{v+p y_{2}}{p},
\end{array}
$$

so that the equations (25) read

$$
\begin{aligned}
\frac{\partial^{2} \xi}{\partial y_{1} \partial y_{1}} & =0 \\
\frac{\partial^{2} \xi}{\partial y_{1} \partial y_{2}} & =0 \\
\frac{\partial^{2} \xi}{\partial y_{1} \partial y_{3}} & =\frac{\partial \xi}{\partial y_{2}} \\
\frac{\partial^{2} \xi}{\partial y_{2} \partial y_{2}} & =0 \\
\frac{\partial^{2} \xi}{\partial y_{2} \partial y_{3}} & =\frac{\partial \xi}{\partial y_{1}} \\
\frac{\partial^{2} \xi}{\partial y_{3} \partial y_{3}} & =\left(\frac{-g+p y_{1}}{p}\right) \frac{\partial \xi}{\partial y_{1}}+\left(\frac{v+p y_{2}}{p}\right) \frac{\partial \xi}{\partial y_{2}} .
\end{aligned}
$$

From (28) and (29) we get

$$
\xi=f\left(y_{3}\right) y_{1}+h\left(y_{2}, y_{3}\right)
$$

and (31) and (30) imply

$$
h\left(y_{2}, y_{3}\right)=f^{\prime}\left(y_{3}\right) y_{2}+b\left(y_{3}\right) .
$$

The equation (32) gives

$$
f\left(y_{3}\right)=c e^{y_{3}}+d e^{-y_{3}}
$$

and from (33) we get the equation for the function $b$

$$
\frac{d^{2} b}{d y_{3}{ }^{2}}=-\frac{g}{p}\left(c e^{y_{3}}+d e^{-y_{3}}\right)+\frac{v}{p}\left(c e^{y_{3}}-d e^{-y_{3}}\right)
$$

solved by

$$
b\left(y_{3}\right)=-\frac{g}{p}\left(c e^{y_{3}}+d e^{-y_{3}}\right)+\frac{v}{p}\left(c e^{y_{3}}-d e^{-y_{3}}\right)+m y_{3}+n
$$

so that the general solution of the system (28)-(33) is

$$
\xi\left(y_{1}, y_{2}, y_{3}\right)=c\left(y_{1}+y_{2}\right) e^{y_{3}}+d\left(y_{1}-y_{2}\right) e^{-y_{3}}+\frac{c(v-g)}{p} e^{y_{3}}-\frac{d(v+g)}{p} e^{-y_{3}}+m y_{3}+n,
$$

where $m, n, c, d$ are integration constants. As the transformation formulas (25) are the same for all the coordinate components $\xi_{i}$ we can write the flat coordinates in general as

$$
\begin{aligned}
\xi_{1}\left(y_{1}, y_{2}, y_{3}\right) & =c_{1}\left(y_{1}+y_{2}\right) e^{y_{3}}+d_{1}\left(y_{1}-y_{2}\right) e^{-y_{3}}+\frac{c_{1}(v-g)}{p} e^{y_{3}}-\frac{d_{1}(v+g)}{p} e^{-y_{3}} \\
& +m_{1} y_{3}+n_{1}, \\
\xi_{2}\left(y_{1}, y_{2}, y_{3}\right) & =c_{2}\left(y_{1}+y_{2}\right) e^{y_{3}}+d_{2}\left(y_{1}-y_{2}\right) e^{-y_{3}}+\frac{c_{2}(v-g)}{p} e^{y_{3}}-\frac{d_{2}(v+g)}{p} e^{-y_{3}}
\end{aligned}
$$




$$
\begin{aligned}
& +m_{2} y_{3}+n_{2}, \\
\xi_{3}\left(y_{1}, y_{2}, y_{3}\right) & =c_{3}\left(y_{1}+y_{2}\right) e^{y_{3}}+d_{3}\left(y_{1}-y_{2}\right) e^{-y_{3}}+\frac{c_{3}(v-g)}{p} e^{y_{3}}-\frac{d_{3}(v+g)}{p} e^{-y_{3}} \\
& +m_{3} y_{3}+n_{3} .
\end{aligned}
$$

and the integration constants will be determined by the required form of the constant metric. When we choose

$$
\left[\frac{\partial \xi_{k}}{\partial y_{i}}\right]_{\vec{y}=\overrightarrow{0}}=\delta_{i}^{k}
$$

then

$$
\begin{aligned}
& \xi_{1}\left(y_{1}, y_{2}, y_{3}\right)=y_{1} \cosh \left(y_{3}\right)+y_{2} \sinh \left(y_{3}\right)+\frac{v}{p} \sinh \left(y_{3}\right)-\frac{g}{p} \cosh \left(y_{3}\right)-\frac{v}{p} y_{3}+n_{1} \\
& \xi_{2}\left(y_{1}, y_{2}, y_{3}\right)=y_{1} \sinh \left(y_{3}\right)+y_{2} \cosh \left(y_{3}\right)+\frac{v}{p} \cosh \left(y_{3}\right)-\frac{g}{p} \sinh \left(y_{3}\right)+\frac{g}{p} y_{3}+n_{2} \\
& \xi_{3}\left(y_{1}, y_{2}, y_{3}\right)=y_{3}+n_{3}
\end{aligned}
$$

and

$$
\tilde{G}(\xi)=\left(\begin{array}{ccc}
p & 0 & v \\
0 & -p & g \\
v & g & r
\end{array}\right) .
$$

This constant form can be transformed by linear transformation

$$
\begin{aligned}
& y_{1}^{\prime}=(\sqrt{|p|}) \xi_{1}+\varepsilon\left(\frac{v}{\sqrt{|p|}}\right) \xi_{3}, \\
& y_{2}^{\prime}=(\sqrt{|p|}) \xi_{2}-\varepsilon\left(\frac{g}{\sqrt{|p|}}\right) \xi_{3}, \\
& y_{3}^{\prime}=\left(\sqrt{\left|r+\left(\frac{\varepsilon g^{2}}{|p|}-\frac{\varepsilon v^{2}}{|p|}\right)\right|}\right) \xi_{3},
\end{aligned}
$$

where

$$
\begin{aligned}
\varepsilon & =\left\{\begin{aligned}
1 & \text { for } p>0 \\
-1 & \text { for } p<0,
\end{aligned}\right. \\
\lambda & =\left\{\begin{aligned}
1 & \text { for }\left(r+\frac{\varepsilon g^{2}}{|p|}-\frac{\varepsilon v^{2}}{|p|}\right)>0 \\
-1 & \text { for }\left(r+\frac{\varepsilon g^{2}}{|p|}-\frac{\varepsilon v^{2}}{|p|}\right)<0
\end{aligned}\right.
\end{aligned}
$$

(for $p=0$ or $r+\frac{g^{2}}{p}-\frac{v^{2}}{p}=0$ the metric is not invertible) to the diagonal form

$$
G^{\prime}\left(y^{\prime}\right)=\left(\begin{array}{ccc}
\varepsilon & 0 & 0 \\
0 & -\varepsilon & 0 \\
0 & 0 & \lambda
\end{array}\right) .
$$

The solution of other cases is a bit more complicated, nevertheless, we were able to find the flat coordinates in all investigated cases. Results are given below. 


\subsection{Solution for the $\sigma-$ model $(2 \mid 1)$}

The nonzero components of the affine connection for the metric (13) are

$$
\begin{array}{llll}
\Gamma_{22}^{1}=\frac{-u^{2} g-u^{3} y_{2}+u v q}{u^{2} r-2 u v g+v^{2} q}, & \Gamma_{23}^{1}=\frac{-v u g-u^{2} v y_{2}+v^{2} q}{u^{2} r-2 u v g}, & \Gamma_{33}^{1}=\frac{-v u r-u v^{2} y_{2}+v^{2} g}{u^{2} r-2 u v g}, \\
\Gamma_{22}^{2}=\frac{-u^{2} v}{u^{2} r-2 u v g+v^{2} q}, & \Gamma_{23}^{2}=\frac{-u v^{2} q}{u^{2} r-2 u v g+v^{2} q}, & \Gamma_{33}^{2}=\frac{-v^{3}}{u^{2} r-2 u v g+v^{2} q} \\
\Gamma_{22}^{3}=\frac{u^{3}}{u^{2} r-2 u v g+v^{2} q}, & \Gamma_{23}^{3}=\frac{u^{2} v}{u^{2} r-2 u v g+v^{2} q}, & \Gamma_{33}^{3}=\frac{u v^{2}}{u^{2} r-2 u v g+v^{2} q}
\end{array}
$$

The general solution of the equations (25) is

$$
\begin{aligned}
\xi\left(y_{1}, y_{2}, y_{3}\right)= & a-6 d(u \rho-v \omega)^{2} y_{1}+b Y+c Y^{2}+d(u \rho-v \omega) Y^{3}+ \\
& (2 c-6 d \rho \omega) Z+3 d u v Z^{2}-6 d v \omega Y Z
\end{aligned}
$$

where $a, b, c, d$ are integration constants,

$$
\begin{gathered}
Y=u y_{2}+v y_{3}, \quad Z=\omega y_{2}+\rho y_{3}, \\
\omega=g u-q v, \quad \rho=r u-g v .
\end{gathered}
$$

When we choose the initial conditions (26) then the flat coordinates in terms of the group coordinates are

$$
\begin{aligned}
\xi_{1}\left(y_{1}, y_{2}, y_{3}\right)= & \frac{\left(6 y_{1} u^{2} r-12 y_{1} u v g+6 y_{1} v^{2} q-3 u^{2} y_{2}^{2} g+3 u y_{2}^{2} v q-u^{3} y_{2}^{3}\right)}{6\left(u^{2} r-2 u v g+v^{2} q\right)} \\
& +\frac{\left(-3 u^{2} v y_{3} y_{2}^{2}-3 u y_{2} v^{2} y_{3}^{2}-6 u y_{2} v y_{3} g+6 y_{2} v^{2} y_{3} q-v^{3} y_{3}^{3}\right)}{6\left(u^{2} r-2 u v g+v^{2} q\right)} \\
& +\frac{\left(v^{2} y_{3}^{2} g-v y_{3}^{2} u r\right)}{2\left(u^{2} r-2 u v g+v^{2} q\right)}+d_{1}, \\
\xi_{2}\left(y_{1}, y_{2}, y_{3}\right)= & \frac{\left(-u^{2} v y_{2}^{2}-2 u v^{2} y_{3} y_{2}+2 r u^{2} y_{2}-4 u v y_{2} g+2 y_{2} v^{2} q-v^{3} y_{3}{ }^{2}\right)}{2\left(u^{2} r-2 u v g+v^{2} q\right)}+d_{2}, \\
\xi_{3}\left(y_{1}, y_{2}, y_{3}\right)= & \frac{\left(u^{3} y_{2}^{2}+2 u^{2} v y_{3} y_{2}+u v^{2} y_{3}^{2}+2 y_{3} u^{2} r-4 v y_{3} u g+2 v^{2} y_{3} q\right)}{2\left(u^{2} r-2 u v g+v^{2} q\right)}+d_{3} .
\end{aligned}
$$

and

$$
\widetilde{G}(\xi)=\left(\begin{array}{ccc}
0 & u & v \\
u & q & g \\
v & g & r
\end{array}\right) .
$$

By the linear transformation

$$
\begin{aligned}
y_{1}^{\prime} & =\left(\frac{u}{\varepsilon \sqrt{|q|}}\right) \xi_{1}+(\sqrt{|q|}) \xi_{2}+\left(\frac{g}{\varepsilon \sqrt{|q|}}\right) \xi_{3}, \\
y_{2}^{\prime} & =\left(\frac{u}{\sqrt{|q|}}\right) \xi_{1}+\left(\left(\frac{u g}{q}-\frac{v}{\varepsilon}\right)\left(\frac{\sqrt{|q|}}{u}\right)\right) \xi_{3}, \\
y_{3}^{\prime} & =\sqrt{\left|\left(\frac{g u}{|q|}-\frac{v}{\varepsilon}\right)^{2} \frac{|q|}{u^{2}}-\frac{\varepsilon g^{2}}{|q|}+\frac{r}{\varepsilon}\right|} \xi_{3},
\end{aligned}
$$

where

$$
\varepsilon=\left\{\begin{aligned}
1 & \text { for } q>0 \\
-1 & \text { for } q<0
\end{aligned}\right.
$$




$$
\lambda=\left\{\begin{array}{rc}
1 & \text { for }\left(\left(\frac{g u}{|q|}-\frac{v}{\varepsilon}\right)^{2} \frac{|q|}{u^{2}}-\frac{\varepsilon g^{2}}{|q|}+\frac{r}{\varepsilon}\right)>0 \\
-1 & \text { for }\left(\left(\frac{g u}{|q|}-\frac{v}{\varepsilon}\right)^{2} \frac{|q|}{u^{2}}-\frac{\varepsilon g^{2}}{|q|}+\frac{r}{\varepsilon}\right)<0
\end{array}\right.
$$

we can transform the metric tensor (13) to the constant diagonal form

$$
G^{\prime}\left(y^{\prime}\right)=\left(\begin{array}{ccc}
\varepsilon & 0 & 0 \\
0 & -\varepsilon & 0 \\
0 & 0 & \lambda \varepsilon
\end{array}\right) .
$$

\subsection{Solution for the $\sigma-\operatorname{model}(3 \mid 1)$}

The nonzero components of the affine connection for the metric (14) are

$$
\Gamma_{11}^{1}=-2, \quad \Gamma_{11}^{2}=\frac{\left(p q-u^{2}\right) e^{2 y_{1}}+z u}{q z}, \quad \Gamma_{11}^{3}=\frac{\left(p q-u^{2}\right) e^{2 y_{1}}-z u}{q z} .
$$

The general solution of the equations (25) is

$$
\xi\left(y_{1}, y_{2}, y_{3}\right)=c y_{3}+a y_{2}+\frac{u(a-c)}{2 q} y_{1}+\frac{\left(p q-u^{2}\right)(a+c)}{8 q z} e^{2 y_{1}}+d e^{-2 y_{1}}+b,
$$

where $a, b, c, d$ are integration constants. When we choose the initial conditions (26) then the flat coordinates in terms of the group coordinates are

$$
\begin{aligned}
\xi_{1}\left(y_{1}, y_{2}, y_{3}\right)= & -\frac{1}{2} e^{-2 y_{1}}+b_{1} \\
\xi_{2}\left(y_{1}, y_{2}, y_{3}\right)= & y_{2}+\frac{u}{2 q} y_{1}+\frac{\left(p q-u^{2}\right)}{8 q z} e^{2 y_{1}}+\frac{\left(p q-u^{2}+2 u z\right)}{8 q z} e^{-2 y_{1}}, \\
& +b_{2}, \\
\xi_{3}\left(y_{1}, y_{2}, y_{3}\right)= & y_{3}-\frac{u}{2 q} y_{1}+\frac{\left(p q-u^{2}\right)}{8 q z} e^{2 y_{1}}+\frac{\left(p q-u^{2}-2 u z\right)}{8 q z} e^{-2 y_{1}} \\
& +b_{3},
\end{aligned}
$$

and

$$
\widetilde{G}(\xi)=\left(\begin{array}{ccc}
p & u+z & z-u \\
u+z & q & -q \\
z-u & -q & q
\end{array}\right) .
$$

By the linear transformation

$$
\begin{aligned}
& y_{1}^{\prime}=(\sqrt{|p|}) \xi_{1}+\left(\frac{u+z}{\varepsilon \sqrt{|p|}}\right) \xi_{2}+\left(\frac{z-u}{\varepsilon \sqrt{|p|}}\right) \xi_{3}, \\
& y_{2}^{\prime}=\left(\sqrt{\left|\frac{(u+z)^{2}}{|p|}-\frac{q}{\varepsilon}\right|}\right) \xi_{2}+\left(\frac{\frac{q}{\delta \varepsilon}+\frac{\left(z^{2}-u^{2}\right)}{\delta|p|}}{\sqrt{\left|\frac{(u+z)^{2}}{|p|}-\frac{q}{\varepsilon}\right|}}\right) \xi_{3}, \\
& y_{3}^{\prime}=\left(\sqrt{\left|\frac{\varepsilon \delta\left(\frac{\left(z^{2}-u^{2}\right)}{\delta|p|}+\frac{q}{\delta \varepsilon}\right)^{2}}{\left(\frac{(u+z)^{2}}{|p|}-\frac{q}{\varepsilon}\right)}-\frac{(z-u)^{2}}{\varepsilon|p|}+q\right|}\right) \xi_{3},
\end{aligned}
$$

where

$$
\varepsilon=\left\{\begin{aligned}
1 & \text { for } p>0 \\
-1 & \text { for } p<0
\end{aligned}\right.
$$




$$
\begin{aligned}
& \delta=\left\{\begin{aligned}
1 & \text { for }\left(\frac{(u+z)^{2}}{|p|}-\frac{q}{\varepsilon}\right)>0 \\
-1 & \text { for }\left(\frac{(u+z)^{2}}{|p|}-\frac{q}{\varepsilon}\right)<0,
\end{aligned}\right. \\
& \lambda=\left\{\begin{aligned}
1 & \text { for }\left(\varepsilon \delta \frac{\left(\frac{z^{2}-u^{2}}{\delta p \mid}+\frac{q}{\delta \varepsilon}\right)^{2}}{\left(\frac{u+z)^{2}}{p \mid}-\frac{q}{\varepsilon}\right)}-\frac{(z-u)^{2}}{\varepsilon|p|}+q\right)>0 \\
-1 & \text { for }\left(\varepsilon \delta \frac{\left(\frac{z^{2}-u^{2}}{\delta|p|}+\frac{q}{\delta \varepsilon}\right)^{2}}{\left(\frac{(u+z)^{2}}{|p|}-\frac{q}{\varepsilon}\right)}-\frac{(z-u)^{2}}{\varepsilon|p|}+q\right)<0
\end{aligned}\right.
\end{aligned}
$$

we can transform the metric tensor (14) to the constant diagonal form

$$
G^{\prime}\left(y^{\prime}\right)=\left(\begin{array}{ccc}
\varepsilon & 0 & 0 \\
0 & -\varepsilon \delta & 0 \\
0 & 0 & \lambda
\end{array}\right) .
$$

\subsection{Solution for the $\sigma$-model $(4 \mid 1)$}

The nonzero components of the affine connection for the metric (15) are

$$
\begin{array}{lll}
\Gamma_{11}^{1}=-1 & \Gamma_{11}^{2}=\frac{v}{q} e^{y_{1}} & \Gamma_{12}^{2}=-1 \\
\Gamma_{21}^{2}=-1 & \Gamma_{11}^{3}=\left(\frac{p}{v}-\frac{u}{q}-\frac{v}{q} y_{1}\right) e^{y_{1}} & \Gamma_{12}^{3}=\frac{u}{v}+y_{1} \\
\Gamma_{22}^{3}=\frac{q}{v} e^{-y_{1}} & &
\end{array}
$$

The general solution of the equations (25) is

$$
\begin{aligned}
\xi\left(y_{1}, y_{2}, y_{3}\right)= & c y_{3}+\frac{q c}{2 v} y_{2}^{2} e^{-y 1}+a y_{2} e^{-y_{1}}+c y_{1} y_{2}+\frac{c u}{v} y_{2}-c y_{2}+\frac{a v}{q} y_{1} \\
& +\frac{p c}{2 v} e^{y_{1}}+\frac{c v}{2 q} e^{y_{1}}+d e^{-y_{1}}+b
\end{aligned}
$$

where $a, b, c, d$ are integration constants. When we choose the initial conditions (26) then the flat coordinates in terms of the group coordinates are

$$
\begin{aligned}
\xi_{1}\left(y_{1}, y_{2}, y_{3}\right)= & -e^{-y_{1}} \\
\xi_{2}\left(y_{1}, y_{2}, y_{3}\right)= & y_{2} e^{-y_{1}}+\frac{v}{q} y_{1}+\frac{v}{q} e^{-y_{1}} \\
\xi_{3}\left(y_{1}, y_{2}, y_{3}\right)= & \frac{(p q-2 u v)}{2 q v} e^{-y_{1}}+\frac{v}{2 q} e^{-y_{1}}+y_{3}+\frac{q}{2 v} y_{2}^{2} e^{-y_{1}}+y_{1} y_{2}+\frac{u}{v} y_{2} \\
& -y_{2}+\frac{p}{2 v} e^{y_{1}}-\frac{u}{v} y_{2} e^{-y_{1}}+y_{2} e^{-y_{1}}+\frac{(v-u)}{q} y_{1}-\frac{v}{2 q} e^{y_{1}} .
\end{aligned}
$$

and

$$
\widetilde{G}(\xi)=\left(\begin{array}{ccc}
p & u & v \\
u & q & 0 \\
v & 0 & 0
\end{array}\right) .
$$

By the linear transformation

$$
\begin{aligned}
& y_{1}^{\prime}=\sqrt{|q|} \xi_{2}+\frac{\varepsilon u}{\sqrt{|q|}} \xi_{1}, \\
& y_{2}^{\prime}=\sqrt{\left|p-\frac{\varepsilon u^{2}}{|q|}\right|} \xi_{1}+\left(\frac{\delta v}{\sqrt{\left|p-\frac{\varepsilon u^{2}}{|q|}\right|}}\right) \xi_{3},
\end{aligned}
$$




$$
y_{3}^{\prime}=\left(\sqrt{\left|\frac{\delta v^{2}}{\left|p-\frac{\varepsilon u^{2}}{|q|}\right|}\right|}\right) \xi_{3},
$$

where

$$
\begin{aligned}
& \varepsilon=\left\{\begin{aligned}
1 & \text { for } q>0 \\
-1 & \text { for } q<0,
\end{aligned}\right. \\
& \delta=\left\{\begin{aligned}
1 & \text { for }\left(p-\frac{\varepsilon u^{2}}{|q|}\right)>0 \\
-1 & \text { for }\left(\mid p-\frac{\varepsilon u^{2}}{|q|}\right)<0,
\end{aligned}\right. \\
& \lambda=\left\{\begin{aligned}
1 & \text { for }\left(\frac{\delta v^{2}}{\left.\mid p-\frac{\varepsilon u^{2}}{|q|}\right)>0}\right. \\
-1 & \text { for }\left(\frac{\delta v^{2}}{\left.\mid p-\frac{\varepsilon u^{2}}{|q|}\right)}<0\right.
\end{aligned}\right.
\end{aligned}
$$

we can transform the metric tensor (15) to the constant diagonal form

$$
G^{\prime}\left(y^{\prime}\right)=\left(\begin{array}{ccc}
\varepsilon & 0 & 0 \\
0 & \delta & 0 \\
0 & 0 & -\lambda
\end{array}\right) .
$$

\subsection{Solution for the $\sigma-$ model $(5 \mid 1)$}

The nonzero components of the affine connection for the metric (16) are

$$
\begin{array}{lll}
\Gamma_{11}^{1}=-1 & \Gamma_{11}^{2}=\frac{r p}{(u r-v g)} e^{y_{1}} & \Gamma_{12}^{2}=\frac{g v}{(u r-v g)} \\
\Gamma_{13}^{2}=\frac{v r}{(u r-v g)} & \Gamma_{22}^{2}=\frac{g^{2}}{(u r-v g)} e^{-y_{1}} & \Gamma_{23}^{2}=\frac{g r}{(u r-v g)} e^{-y_{1}} \\
\Gamma_{33}^{2}=\frac{r^{2}}{(u r-v g)} e^{-y_{1}} & \Gamma_{11}^{3}=-\frac{p g}{(u r-v g)} e^{y_{1}} & \Gamma_{21}^{3}=-\frac{u g}{(u r-v g)} \\
\Gamma_{22}^{3}=-\frac{g^{3}}{r(u r-v g)} e^{-y_{1}} & \Gamma_{31}^{3}=-\frac{u r}{(u r-v g)} & \Gamma_{32}^{3}=-\frac{g^{2}}{(u r-v g)} e^{-y_{1}} \\
\Gamma_{33}^{3}=-\frac{g r}{(u r-v g)} e^{-y_{1}} . & &
\end{array}
$$

The general solution of the equations (25) is

$$
\frac{1}{2} a p e^{y_{1}}+a\left(u y_{2}+v y_{3}\right)+e^{-y_{1}}\left(\frac{\left(g y_{2}+r y_{3}\right)\left(2 c r+a r y_{3}+a g y_{2}\right)}{2 r}-b\right)+d
$$

where $a, b, c, d$ are integration constants. When we choose the initial conditions (26) then the flat coordinates in terms of the group coordinates are

$$
\begin{aligned}
& \xi_{1}\left(y_{1}, y_{2}, y_{3}\right)=-e^{-y_{1}}+d_{1} \\
& \xi_{2}\left(y_{1}, y_{2}, y_{3}\right)=\frac{p r \cosh y_{1}+r\left(u y_{2}+v y_{3}\right)+e^{-y_{1}}\left(\frac{1}{2}\left(g y_{2}+r y_{3}\right)^{2}-v\left(g y_{2}+r y_{3}\right)\right)}{r u-g v}+d_{2} \\
& \xi_{3}\left(y_{1}, y_{2}, y_{3}\right)=\frac{-p g \cosh y_{1}+g\left(u y_{2}+v y_{3}\right)+e^{-y_{1}}\left(-\frac{g}{2 r}\left(g y_{2}+r y_{3}\right)^{2}+u\left(g y_{2}+r y_{3}\right)\right)}{r u-g v}+d_{3}
\end{aligned}
$$

and

$$
\widetilde{G}(\xi)=\left(\begin{array}{ccc}
p & u & v \\
u & \frac{g^{2}}{r} & g \\
v & g & r
\end{array}\right) .
$$


By the linear transformation

$$
\begin{aligned}
& y_{1}^{\prime}=(\sqrt{|p|}) \xi_{1}+\left(\frac{u}{\varepsilon \sqrt{|p|}}\right) \xi_{2}+\left(\frac{v}{\varepsilon \sqrt{|p|}}\right) \xi_{3}, \\
& y_{2}^{\prime}=\left(\sqrt{\left|\frac{u^{2}}{|p|}-\frac{g^{2}}{\varepsilon r}\right|}\right) \xi_{2}+\left(\frac{\frac{v u}{\delta|p|}-\frac{g}{\delta \varepsilon}}{\sqrt{\left|\frac{u^{2}}{|p|}-\frac{g^{2}}{\varepsilon r}\right|}}\right) \xi_{3}, \\
& y_{3}^{\prime}=\left(\sqrt{\left.\left|r-\frac{v^{2}}{\varepsilon|p|}+\frac{\varepsilon \delta\left(\frac{v u}{|p|}-g \varepsilon\right)^{2} \mid}{\left|\frac{u^{2}}{|p|}-\frac{g^{2}}{\varepsilon r}\right|}\right|\right) \xi_{3},},\right.
\end{aligned}
$$

where

$$
\begin{aligned}
& \varepsilon=\left\{\begin{aligned}
1 & \text { for } p>0 \\
-1 & \text { for } p<0,
\end{aligned}\right. \\
& \delta=\left\{\begin{aligned}
1 & \text { for }\left(\frac{u^{2}}{|p|}-\frac{g^{2}}{\varepsilon r}\right)>0 \\
-1 & \text { for }\left(\frac{u^{2}}{|p|}-\frac{g^{2}}{\varepsilon r}\right)<0,
\end{aligned}\right. \\
& \lambda=\left\{\begin{aligned}
1 & \text { for }\left(r-\frac{v^{2}}{\varepsilon|p|}+\frac{\varepsilon \delta\left(\frac{v u}{|p|}-g \varepsilon\right)^{2}}{\left|\frac{u^{2}}{|p|}-\frac{g^{2}}{\varepsilon r}\right|}\right)>0 \\
-1 & \text { for }\left(r-\frac{v^{2}}{\varepsilon|p|}+\frac{\varepsilon \delta\left(\frac{v u}{\mid p}-g \varepsilon\right)^{2}}{\left|\frac{u^{2}}{|p|}-\frac{g^{2}}{\varepsilon r}\right|}\right)<0
\end{aligned}\right.
\end{aligned}
$$

we can transform the metric tensor (16) to the constant diagonal form

$$
G^{\prime}\left(y^{\prime}\right)=\left(\begin{array}{ccc}
\varepsilon & 0 & 0 \\
0 & -\delta \varepsilon & 0 \\
0 & 0 & \lambda
\end{array}\right) .
$$

\subsection{Solution for the $\sigma$-model $\left(7_{0} \mid 1\right)$}

The nonzero components of the affine connection for this metric are

$$
\begin{aligned}
& \Gamma_{23}^{1}=1 \quad \Gamma_{32}^{1}=1 \quad \Gamma_{33}^{1}=\frac{g}{p}-y_{1} \\
& \Gamma_{13}^{2}=-1 \Gamma_{31}^{2}=-1 \Gamma_{33}^{2}=-\frac{z}{p}-y_{2} .
\end{aligned}
$$

The general solution of the equations (25) is

$$
\xi\left(y_{1}, y_{2}, y_{3}\right)=\left(\frac{g}{p}-y_{1}\right)\left(i a e^{i y_{3}}-i b e^{-i y_{3}}\right)-\left(\frac{z}{p}+y_{2}\right)\left(a e^{i y_{3}}+b e^{-i y_{3}}\right)+c y_{3}+d,
$$

where $a, b, c, d$ are integration constants. When we choose the initial conditions (26) then the flat coordinates in terms of the group coordinates are

$$
\begin{aligned}
& \xi_{1}\left(y_{1}, y_{2}, y_{3}\right)=\left(-\frac{g}{p}+y_{1}\right) \cos \left(y_{3}\right)+\left(\frac{z}{p}+y_{2}\right) \sin \left(y_{3}\right)-\frac{z}{p} y_{3}+d_{1} \\
& \xi_{2}\left(y_{1}, y_{2}, y_{3}\right)=\left(\frac{g}{p}-y_{1}\right) \sin \left(y_{3}\right)+\left(\frac{z}{p}+y_{2}\right) \cos \left(y_{3}\right)-\frac{g}{p} y_{3}+d_{2} \\
& \xi_{3}\left(y_{1}, y_{2}, y_{3}\right)=y_{3}+d_{3} .
\end{aligned}
$$


and

$$
\widetilde{G}(\xi)=\left(\begin{array}{ccc}
p & 0 & z \\
0 & p & g \\
z & g & r
\end{array}\right)
$$

By the linear transformation

$$
\begin{aligned}
& \tilde{y}_{1}=(\sqrt{|p|}) \xi_{1}+\frac{\varepsilon z}{\sqrt{|p|}} \xi_{3}, \\
& \tilde{y}_{2}=(\sqrt{|p|}) \xi_{2}+\frac{\varepsilon g}{\sqrt{|p|}} \xi_{3}, \\
& \tilde{y}_{3}=\left(\sqrt{\left|r-\frac{\varepsilon z^{2}}{|p|}-\frac{\varepsilon g^{2}}{|p|}\right|}\right) \xi_{3},
\end{aligned}
$$

where

$$
\begin{gathered}
\varepsilon=\left\{\begin{aligned}
1 & \text { for } p>0 \\
-1 & \text { for } p<0,
\end{aligned}\right. \\
\lambda=\left\{\begin{aligned}
1 & \text { for }\left(r-\frac{\varepsilon z^{2}}{|p|}-\frac{\varepsilon g^{2}}{|p|}\right)>0 \\
-1 & \text { for }\left(r-\frac{\varepsilon z^{2}}{|p|}-\frac{\varepsilon g^{2}}{|p|}\right)<0
\end{aligned}\right.
\end{gathered}
$$

we can transform the metric tensor (18) to the constant diagonal form

$$
G^{\prime}(\tilde{y})=\left(\begin{array}{ccc}
\varepsilon & 0 & 0 \\
0 & \varepsilon & 0 \\
0 & 0 & \lambda
\end{array}\right) .
$$

\subsection{Solution for the $\sigma-$ model $\left(1 \mid 6_{0}\right)$}

The nonzero components of the affine connection for the metric (19) are

$$
\begin{aligned}
& \Gamma_{11}^{1}=-\frac{\left(1+k y_{1}\right) k}{\left(1+2 k y_{1}+k^{2} y_{1}{ }^{2}-k^{2} y_{2}{ }^{2}\right)} \quad \Gamma_{21}^{1}=\frac{k^{2} y_{2}}{\left(1+2 k y_{1}+k^{2} y_{1}{ }^{2}-k^{2} y_{2}{ }^{2}\right)} \\
& \Gamma_{22}^{1}=-\frac{\left(1+k y_{1}\right) k}{\left(1+2 k y_{1}+k^{2} y_{1}{ }^{2}-k^{2} y_{2}{ }^{2}\right)} \quad \Gamma_{22}^{2}=\frac{k^{2} y_{2}}{\left(1+2 k y_{1}+k^{2} y_{1}{ }^{2}-k^{2} y_{2}{ }^{2}\right)} \\
& \Gamma_{11}^{2}=\frac{k^{2} y_{2}}{\left(1+2 k y_{1}+k^{2} y_{1}{ }^{2}-k^{2} y_{2}{ }^{2}\right)} \quad \Gamma_{12}^{2}=-\frac{\left(1+k y_{1}\right) k}{\left(1+2 k y_{1}+k^{2} y_{1}{ }^{2}-k^{2} y_{2}{ }^{2}\right)} \\
& \Gamma_{11}^{3}=\frac{\left(1+k y_{1}\right) k q y_{1} y_{2}}{\left(1+2 k y_{1}+k^{2} y_{1}{ }^{2}-k^{2} y_{2}{ }^{2}\right)} \quad \Gamma_{21}^{3}=-\frac{k^{2} q y_{1} y_{2}}{\left(1+2 k y_{1}+k^{2} y_{1}{ }^{2}-k^{2} y_{2}{ }^{2}\right)} \\
& \Gamma_{22}^{3}=\frac{q\left(-1+k^{2} y_{2}{ }^{2}-k y_{1}\right)}{\left(1+2 k y_{1}+k^{2} y_{1}{ }^{2}-k^{2} y_{2}{ }^{2}\right)}
\end{aligned}
$$

The general solution of the equations (25) is

$$
\begin{aligned}
\xi\left(y_{1}, y_{2}, y_{3}\right)= & \frac{(q a+4 k b)}{4 k^{2}} \ln \left(1+k\left(y_{1}-y_{2}\right)\right)+\frac{(q a+4 k c)}{4 k^{2}} \ln \left(1+k\left(y_{1}+y_{2}\right)\right) \\
& -\frac{q a}{2 k} y_{1}+\frac{1}{4}\left(q a\left(y_{1}^{2}-y_{2}^{2}\right)\right)+a y_{3}+d .
\end{aligned}
$$

where $a, b, c, d$ are integration constants. When we choose the initial conditions (26) then the flat coordinates in terms of the group coordinates are

$$
\begin{aligned}
\xi_{1}\left(y_{1}, y_{2}, y_{3}\right)= & \frac{1}{2 k} \ln \left(1+k\left(y_{1}-y_{2}\right)\right)+\frac{1}{2 k} \ln \left(1+k\left(y_{1}+y_{2}\right)\right)+d_{1} \\
\xi_{2}\left(y_{1}, y_{2}, y_{3}\right)= & -\frac{1}{2 k} \ln \left(1+k\left(y_{1}-y_{2}\right)\right)+\frac{1}{2 k} \ln \left(1+k\left(y_{1}+y_{2}\right)\right)+d_{2} \\
\xi_{3}\left(y_{1}, y_{2}, y_{3}\right)= & \frac{q}{4 k^{2}} \ln \left(1+k\left(y_{1}-y_{2}\right)\right)+\frac{q}{4 k^{2}} \ln \left(1+k\left(y_{1}+y_{2}\right)\right)-\frac{q}{2 k} y_{1} \\
& +\frac{q}{4}\left(y_{1}^{2}-y_{2}^{2}\right)+y_{3}+d_{3} .
\end{aligned}
$$


and

$$
\widetilde{G}(\xi)=\left(\begin{array}{ccc}
0 & 0 & -k \\
0 & q & 0 \\
-k & 0 & 0
\end{array}\right)
$$

By the linear transformation

$$
\begin{aligned}
& y_{1}^{\prime}=\left(\frac{1}{2} \sqrt{|2 k|}\right) \xi_{1}+\left(\frac{1}{2} \sqrt{|2 k|}\right) \xi_{3}, \\
& y_{2}^{\prime}=(\sqrt{|q|}) \xi_{2}, \\
& y_{3}^{\prime}=\left(\frac{1}{2} \sqrt{|2 k|}\right) \xi_{1}-\left(\frac{1}{2} \sqrt{|2 k|}\right) \xi_{3},
\end{aligned}
$$

where

$$
\begin{gathered}
\varepsilon=\left\{\begin{aligned}
1 & \text { for } k>0 \\
-1 & \text { for } k<0,
\end{aligned}\right. \\
\delta=\left\{\begin{aligned}
1 & \text { for } q>0 \\
-1 & \text { for } q<0
\end{aligned}\right.
\end{gathered}
$$

we can transform the metric tensor (19) to the constant diagonal form

$$
G^{\prime}\left(y^{\prime}\right)=\left(\begin{array}{ccc}
-\varepsilon & 0 & 0 \\
0 & \delta & 0 \\
0 & 0 & \varepsilon
\end{array}\right) .
$$

\subsection{Solution for the $\sigma-\operatorname{model}\left(5 i i \mid 6_{0}\right)$}

The general solution of the equations (25) is

$$
\begin{aligned}
\xi\left(y_{1}, y_{2}, y_{3}\right) & =-\frac{\left(2 e^{y_{2}} q w a+(q(1+2 w) a+2 w b) y_{2}+e^{-\left(y_{1}+y_{2}\right)} q a(2-w)\right)}{4 w^{2}} \\
& -\frac{\left(e^{y_{1}+y_{2}} q w^{2} a\right)}{4 w^{2}}+\frac{\left(q a\left(w^{2}-1\right)+4 w c\right) \ln \left(1+\left(-1+e^{-\left(y_{1}+y_{2}\right)}\right)\right)}{4 w^{2}} \\
& +\frac{(q a(1+2 w)+2 w b) \ln \left(-2 w+e^{y_{2}} w+e^{-y_{1}}(1+w)\right)}{4 w^{2}}+a y_{3} \\
& +\frac{(q w a+2 w b)\left(y_{1}+y_{2}\right)}{4 w^{2}}+\frac{e^{y_{1}} q a(1+w)}{2 w}+\frac{q a\left(y_{1}+y_{2}\right)}{4 w^{2}}+d
\end{aligned}
$$

where $a, b, c, d$ are integration constants. When we choose the initial conditions (26) then the flat coordinates in terms of the group coordinates are

$$
\begin{aligned}
\xi_{1}\left(y_{1}, y_{2}, y_{3}\right)= & -\left(\frac{\ln \left(1-w+e^{-(y 1+y 2)} w\right)+\ln \left(-2 w e^{y_{1}}+e^{y_{1}+y_{2}} w+1+w\right)}{2 w}\right)+d_{1} \\
\xi_{2}\left(y_{1}, y_{2}, y_{3}\right)= & \left(\frac{\ln \left(-2 w e^{y_{1}}+e^{y_{1}+y_{2}} w+1+w\right)-\ln \left(1-w+e^{-\left(y_{1}+y_{2}\right)} w\right)}{2 w}\right)+d_{2} \\
\xi_{3}\left(y_{1}, y_{2}, y_{3}\right)= & -\left(\frac{2 q e^{-y_{2}} w^{2}+q w e^{-\left(y_{1}+y_{2}\right)}-2 q w e^{y_{1}}-q w^{2} e^{-\left(y_{1}+y_{2}\right)}+q w e^{y_{1}+y_{2}}}{4 w^{2}}\right) \\
& +\left(\frac{2 e^{-y_{2}} q w^{2}-q w^{2}+e^{-\left(y_{1}+y_{2}\right)} q \ln \left(1-w+e^{-\left(y_{1}+y_{2}\right)} w\right)}{4 w^{2}}\right) e^{y 1+y^{2}} \\
& +\frac{q}{4 w^{2}} \ln \left(-2 w e^{-y_{2}}+w+e^{-\left(y_{1}+y_{2}\right)}+e^{-\left(y_{1}+y_{2}\right)} w\right)+\frac{q}{4 w^{2}}\left(y_{2}+y_{1}\right) \\
& +y_{3}+d_{3}
\end{aligned}
$$


and

$$
\widetilde{G}(\xi)=\left(\begin{array}{ccc}
0 & 0 & w \\
0 & q & 0 \\
w & 0 & 0
\end{array}\right) .
$$

By the linear transformation

$$
\begin{aligned}
y_{1}^{\prime} & =\frac{1}{2}(\sqrt{2|w|}) \xi_{1}+\frac{1}{2}(\sqrt{2|w|}) \xi_{3}, \\
y_{2}^{\prime} & =(\sqrt{|q|}) \xi_{2} \\
y_{3}^{\prime} & =\frac{1}{2}(\sqrt{2|w|}) \xi_{1}-\frac{1}{2}(\sqrt{2|w|}) \xi_{3}
\end{aligned}
$$

where

$$
\begin{aligned}
& \varepsilon=\left\{\begin{aligned}
1 & \text { for } w>0 \\
-1 & \text { for } w<0
\end{aligned}\right. \\
& \delta=\left\{\begin{aligned}
1 & \text { for } q>0 \\
-1 & \text { for } q<0
\end{aligned}\right.
\end{aligned}
$$

we can transform the metric tensor (21) to the constant diagonal form

$$
G^{\prime}\left(y^{\prime}\right)=\left(\begin{array}{ccc}
\varepsilon & 0 & 0 \\
0 & \delta & 0 \\
0 & 0 & -\varepsilon
\end{array}\right) .
$$

\section{Dilaton fields}

As mentioned in the section 2 the metrics (13) - 18) were obtained in the paper 4 from the requirement that the vanishing $\beta$ equations are satisfied for the constant dilaton field. When we know the flat coordinates of these models we can easily find general forms of the dilaton fields that together with these metrics satisfy the vanishing $\beta$ equations (5)-(7) because in the flat coordinates these equations read

$$
\frac{\partial^{2} \Phi^{\prime}}{\partial y_{i}^{\prime} \partial y_{j}^{\prime}}=0, \quad G^{i j} \frac{\partial \Phi^{\prime}}{\partial y_{i}^{\prime}} \frac{\partial \Phi^{\prime}}{\partial y_{j}^{\prime}}=0 .
$$

The general solution of these equations is

$$
\Phi^{\prime}\left(y_{1}^{\prime}, y_{2}^{\prime}, y_{3}^{\prime}\right)=c_{1} y_{1}^{\prime}+c_{2} y_{2}^{\prime}+c_{3} y_{3}^{\prime}+c_{4}
$$

where $G^{\prime i j} c_{i} c_{j}=0$.

The general form of the dilaton field for the $\sigma$-model $\left(6_{0} \mid 1\right)$ with the metric (17) that follow from (86) and (42) is

$$
\begin{aligned}
\Phi\left(y_{1}, y_{2}, y_{3}\right) & =c_{1}(\sqrt{|p|})\left(y_{1} \cosh \left(y_{3}\right)+y_{2} \sinh \left(y_{3}\right)+\frac{v}{p} \sinh \left(y_{3}\right)-\frac{g}{p} \cosh \left(y_{3}\right)-\frac{v}{p} y_{3}\right) \\
& +c_{1}\left(\frac{\varepsilon v}{\sqrt{|p|}}\right) y_{3} \\
& +c_{2}(\sqrt{|p|})\left(y_{1} \sinh \left(y_{3}\right)+y_{2} \cosh \left(y_{3}\right)+\frac{v}{p} \cosh \left(y_{3}\right)-\frac{g}{p} \sinh \left(y_{3}\right)+\frac{g}{p} y_{3}\right) \\
& -c_{2}\left(\frac{\varepsilon g}{\sqrt{|p|}}\right) y_{3}+c_{3}\left(\sqrt{\left|\varepsilon r+\left(\frac{g^{2}}{p}-\frac{v^{2}}{p}\right)\right|}\right) y_{3}+c_{4}
\end{aligned}
$$


where $\operatorname{sign}(\varepsilon)\left(c_{1}^{2}-c_{2}^{2}\right)+\operatorname{sign}(\varepsilon \delta) c_{3}^{2}=0$.

By a similar way, i.e. as a linear combination of the flat coordinates, we can get the general dilaton fields for the $\sigma$-models with the metrics (13)-(16) and (18). If the metric is positively or negatively definite then the dilaton is constant.

We can also get dilaton fields more general than (20) and (22) for the models $\left(1 \mid 6_{0}\right)$ and $\left(5 i i \mid 6_{0}\right)$. The general form of the dilaton field for the $\sigma$-model $\left(1 \mid 6_{0}\right)$ is

$$
\begin{aligned}
\Phi(y) & =\frac{\sqrt{|2 k|}}{4 k}\left(c_{1}+c_{3}+\left(c_{1}-c_{3}\right) \frac{q}{2 k}\right) \ln \left|\left(1+k\left(y_{1}-y_{2}\right)\right)\left(1+k\left(y_{1}+y_{2}\right)\right)\right| \\
& +\left(c_{1}-c_{3}\right)\left[\frac{1}{2} \sqrt{|2 k|}\left(-\frac{q}{2 k} y_{1}+\frac{q}{4}\left(y_{1}^{2}-y_{2}^{2}\right)+y_{3}\right)\right] \\
& +c_{2} \frac{\sqrt{|q|}}{2 k} \ln \left|\frac{1+k\left(y_{1}+y_{2}\right)}{1+k\left(y_{1}-y_{2}\right)}\right|+c_{4} .
\end{aligned}
$$

where $\operatorname{sign}(q) c_{2}^{2}+\operatorname{sign}(k)\left(c_{3}^{2}-c_{1}^{2}\right)=0$. For special choice of constants $c_{1}=c_{3}=2 k / \sqrt{|2 k|}, c_{2}=$ 0 , we get the dilaton field (20) obtained in [4] by the Poisson-Lie T-duality. The general form of the dilaton field for the $\sigma$-model $\left(5 i i \mid 6_{0}\right)$ can be obtained from (883) as well but it is rather extensive to display.

\section{Conclusions}

We have obtained the explicit transformation between the group coordinates of threedimensional conformal $\sigma$-models living in the flat background and its Riemannian coordinates. The forms of the metric in terms of the group coordinates were found in 4 from requirement of conformal invariance of $\sigma$-models on the solvable Lie groups. The transformations were found by solving the equations that follow from the transformation properties of the Levi-Civita connections. The results can be used for many purposes. Let us mention two of them.

The equations for the dilaton field of the flat $\sigma$-model are easily solvable in the flat coordinates. In the Section 4 we have shown that the coordinate transformations enable us to get the dilaton fields in terms of the group coordinates. These, on the other hand, are convenient for obtaining the dilatons of the conformal $\sigma$-models with nontrivial backgrounds by the Poisson-Lie plurality [6].

Besides that, the fact that all the investigated metrics are flat means that equations of motion of the $\sigma$-models are easily solvable in terms of the flat coordinates and the solutions can be transformed to the group coordinates. The investigated models are Poisson-Lie Tdual or plural to $\sigma$-models with nontrivial backgrounds and it offers a possibility to find classical solutions in the nontrivial background. An example of such solution was given in [7] and other models are being solved now.

\section{References}

[1] C. Klimčík and P. Ševera, Dual non-Abelian duality and the Drinfeld double, Phys. Lett. B 351 (1995) 455, hep-th/9502122.

[2] C. Klimčík, Poisson-Lie T-duality, Nucl. Phys. B (Proc. Suppl.) 46 (1996) 116, hep-th/9509095.

[3] L. P. Eisenhart, Riemannian geometry, Princeton University Press, 1997.

[4] L. Hlavatý and L. Šnobl, Poisson-Lie T-plurality of three-dimensional conformally invariant sigma models II : Nondiagonal metrics and dilaton puzzle, J. High En. Phys. 04:10 (2004) 045, hep-th/0408126. 
[5] L.D. Landau and E.M. Lifshitz, The classical theory of fields, Pergamon Press, London 1987.

[6] R. von Unge, Poisson-Lie T-plurality, J. High En. Phys. 02:07 (2002) 014, hep-th/00205245].

[7] L. Hlavatý, Classical solution of a sigma model in curved background, Phys. Lett. B 625 (2005) 285, hep-th/0506188 